\title{
PRÁCTICAS ECONÓMICAS ALTERNATIVAS, UNA MIRADA DESDE LA TEORÍA FUNDAMENTADA
}

\author{
Eridani Durán Vázquez, Francisco Javier Ruvalcaba Coyaso, Moisés Hussein Chávez Hernández, \\ Bélgica María Romero de Loera y Miguel Ángel Sahagún Padilla \\ Universidad Autónoma de Aguascalientes \\ México
}

\begin{abstract}
RESUMEN
En el siglo XIX surgió la economía social como resultado de la movilización de grupos de obreros que se organizaron para generar alternativas a los trabajos que desempeñaban en una búsqueda para contrarrestar las condiciones de explotación que vivían. De esta manera comenzó una serie de movilizaciones sociales para buscar condiciones laborales más dignas. Aunque las expresiones más conocidas de estos emprendimientos son las cooperativas o mutualidades, hay otros movimientos que se adhieren de manera ideológica a las premisas de la economía social o economías alternativas, como los espacios de trueque y los mercados o tianguis alternativos. En este sentido, el propósito de este trabajo es comprender qué significados les otorgan los participantes a las prácticas de economías alternativas e identificar cómo dichos significados inciden en las prácticas, de manera que han permitido la construcción social de los espacios económicos en la ciudad de Aguascalientes. Se hizo un estudio cualitativo y se desarrolló en dos espacios distintos: 1) tianguis y mercados alternativos, y 2) lugares con prácticas de trueque. Se utiliza como método la teoría fundamentada. Las categorías centrales identificadas son: 1) Alternativo, 2) Perfil de quien participa, 3) Ideología y 4) Activismo. Se observa que estos espacios funcionan sobre todo como una expresión de una adhesión ideológica donde el activismo es parte fundamental de estas prácticas.
\end{abstract}

Palabras Clave:

\section{ALTERNATIVE ECONOMIC PRACTICES, A LOOK FROM GROUNDED THEORY}

\begin{abstract}
The social economy emerged in the 19th century from groups of workers who mobilized to generate other alternatives to work that counteracted the conditions of exploitation that they lived, from that moment a series of social movements began to seek more dignified working conditions. The best-known expressions of these ventures are cooperatives or mutual, however, there are other movements that ideologically adhere to the premises of the Social Economy or Alternative Economies, such as barter spaces and alternative markets or markets. A qualitative study was carried out in the city of Aguascalientes, Mexico, in two different spaces, one of them alternative markets and another dedicated specially to barter practices. Grounded theory was used as a research method. The central categories identified so far are: instrumentalization of labels, profile of who participates, space to spread ideology, indoctrination, alternative and feminism. It is observed that, unlike the studies usually reported in the literature, these spaces are mainly as an expression of an ideological adherence, where activism is a fundamental part of the practices.
\end{abstract}

Keywords:

alternative economies, grounded theory, practices, alternative markets

Bitácora del Artículo:

| Recibido: 21 de Septiembre de 2020 | Aceptado: 07 enero de 2021 | Publicado en línea: Enero - Junio de 2021 | 


\title{
PRÁCTICAS ECONÓMICAS ALTERNATIVAS, UNA MIRADA DESDE LA TEORÍA FUNDAMENTADA
}

\author{
Eridani Durán Vázquez, Francisco Javier Ruvalcaba Coyaso, Moisés Hussein Chávez Hernández, \\ Bélgica María Romero de Loera y Miguel Ángel Sahagún Padilla \\ Universidad Autónoma de Aguascalientes \\ México
}

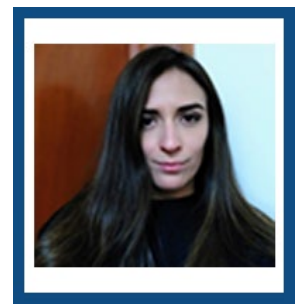

Eridani Durán Vázquez

UAA

Correo:eridani.duran@hotmail.com

Licenciada en Psicología y candidata a maestra en Investigación en Psicología por la Universidad Autónoma de Aguascalientes. Colaboró como profesora adjunta a nivel licenciatura, así como asistente de investigación. Es miembro del Observatorio laboral del estado de Aguascalientes.

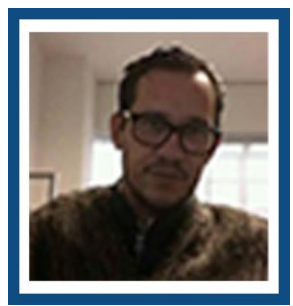

Francisco Javier Ruvalcaba Coyaso UAA

Correo:javier.ruvalcaba@edu.uaa.mx

Licenciado en Psicología, maestría en Psicología del Trabajo y las Organizaciones. doctorado en Psicología con énfasis en Psicología Social y Organizacional. Miembro del Sistema Nacional de Investigadores del 2014 al 2019. Coordinador de diplomados, curso y talleres sobre la norma 035 de riesgos psicosociales en el trabajo.

\section{CONTRIBUCIÓN DE LOS AutORES}

Eridani Durán Vázquez concibió y desarrolló el proyecto | Francisco Javier Ruvalcaba Coyaso hizo revisiones en cuanto a redacción y estilo. Contribuciones en el análisis y conclusiones | Moisés Hussein Chávez Hernández efectuó revisiones conceptuales y aporte en las discusiones | Bélgica María Romero de Loera apoyó en el análisis de datos | Miguel Ángel Sahagún Padilla hizo revisiones en cuanto a redacción y estilo.

\section{AGRADECIMIENTOS}

El artículo fue elaborado como parte de los requisitos para obtener el grado de maestría en Investigación en Psicología de la Universidad Autónoma de Aguascalientes, México.

\section{Datos de FiLIACIÓN DE los Autores}

Eridani Durán Vázquez, Francisco Javier Ruvalcaba Coyaso, Miguel Ángel Sahagún Padilla, Universidad Autónoma de Aguascalientes | Moisés Hussein Chávez Hernández Universidad Autónoma de Baja California | Bélgica María Romero de Loera Universidad Cuauhtémoc

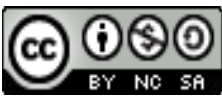

Copyright: (c) 2020 Durán-Vázquez, E.; Ruvalcaba-Coyaso, F.J.; Chávez-Hernández, M.H.; Romero de Loera, B.M.; \& Sahagún-Padilla, M.A. Este es un artículo de acceso abierto distribuido bajo los términos de la licencia Creative Commons Reconocimiento-NoComercial 4.0 Internacional, por lo que su contenido gráfico y escrito se puede compartir, copiar y redistribuir total o parcialmente sin necesidad de permiso expreso de sus autoras con la única condición de que no se puede usar con fines directamente comerciales y los términos legales de cualquier trabajo derivado deben ser los mismos que se expresan en la presente declaración. La única condición es que se cite la fuente con referencia a la Revista Digital Internacional de Psicología y Ciencia Social y a sus autoras. 


\section{TABLA DE CONTENIDO}

MÉTODO

TEORÍA FUNDAMENTADA

Codificación desde la Teoría fundamentada, 27

Codificación abierta, 27

Codificación axial, 27

Codificación selectiva, 27

PROCEDIMIENTO DE ANÁLISIS DE LOS DATOS

REsultados

Discusión

CONCLUSIONES

REFERENCIAS 
D urante el siglo XIX surgen prácticas sociales denominadas como "economías sociales". Nacen como parte de los movimientos efectuados por los obreros que hacían frente a las situaciones de injusticia y sobreexplotación en las fábricas debido al desarrollo industrial (Avendaño, Canales y Atria, 2012; González, 2011). A partir de ahí se inició el desarroIlo de diversas formas de organización económicas que permitían la satisfacción de las necesidades básicas guiadas por otros principios éticos, como la primacía del ser humano sobre el capital, el cuidado del medio ambiente, la inclusión de mujeres en espacios de trabajo y la promoción de una vida digna y sustentable (Monzón-Campos, 2006; Pérez-Mendiguren, Etxezarreta-Etxarri y Guridi-Aldanondo, 2008).

Los términos "economía social", "economía solidaria", "economía social y solidaria", así como "economías alternativas", se usan de manera indiscriminada en la literatura. Estos hacen referencia a las prácticas que se utilizan en contextos de cooperativas, mutualidades, asociaciones y fundaciones, así como mercados alternativos, orgánicos y espacios de trueque (Diez, 2002; Sanz, Carrero y Alcañiz, 2018).

En la revisión de la literatura se logró identificar que usualmente los participantes de estos espacios son individuos que se encuentran en una situación de marginación social (población de escasos recursos económicos, indígenas, migrantes y empleadas domésticas) y con un bajo nivel de escolarización. Así, estos espacios bridan a estos sujetos la posibilidad de tener acceso a una fuente de remuneración, pues dadas sus condiciones están limitados para ingresar de manera exitosa al engranaje del sistema capitalista. No obstante, en la ciudad de Aguascalientes se ha observado la participación de grupos poblacionales que no comparten condiciones de marginación ni de baja escolaridad como los individuos descritos en líneas anteriores (Coutinho, Beiras, Picinin y Lückman, 2005; Calil y Szymanski, 2010; Marañón-Pimentel, 2013; García y Valdés, 2015; Méndez, 2015; Santos, et al., 2016).

Sanz, Carrero y Alcañiz (2018) describen las diferencias entre los participantes de estas economías. Por un lado se encuentran aquellos que utilizan estos espacios de manera instrumental como una forma para crear espacios asistenciales donde se satisfacen algunas necesidades básicas. Por otro lado, hay otros participantes que están por componentes ideológicos que guardan relación con la transformación social. Siguiendo a Sanz, Carrero y Alcañiz (2018) ven en sus prácticas y discursos "[...] la forma de construir «otra economía» de la economía social y solidaria se basa en -y se legitima mediante- un discurso ideológico político-moral en el que se enfatizan las diferencias en su actuación, respecto a una racionalidad capitalista" (p.39). Además, dado que en estos espacios se fomenta el prosumo, es decir el producir y consumir, Duhau y Giglia (2007) afirman que las prácticas de consumo no se realizan sólo de manera instrumental, sino que también están encargadas por una idiosincrasia.

Además de los espacios típicamente conocidos dentro de la economía social solidaria, como las cooperativas, también existen los mercados o tianguis locales en los que se conservan prácticas de comercio antiguas, donde resurgen y adquieren diferentes significados (Roldán, Gracia, Santana y Horbath, 2016). Algunos de ellos autonombrados como mercados alternativos, solidarios u orgánicos, buscan asimismo la venta de productos libres de químicos y la promoción de un vínculo de intercambio directo, ya que son espacios de interacción donde además de favorecer lazos de socialización con los pertenecientes a la región, los sujetos resignifican sus identidades.

Santana (2011) observa en los mercados alternativos una propuesta de economía solidaria, pues percibe que son una forma de combatir el consumismo debido al papel activo que desarrollan sus miembros. Así, estos sujetos pueden acceder al mercado sin entrar en el mercado capitalista debido a que el trabajo es valorado por el consumidor, y por ende se paga un precio justo. Del mismo modo representa una alternativa a los supermercados. No obstante, es importante destacar que, aunque la economía solidaria se plantea como una alternativa al sistema capitalista, las prácticas están envueltas en dicho sistema.

Debido a que la producción académica respecto a las economías sociales y solidarias muestra una tendencia por la construcción continua de definiciones o propuestas de aplicación o mejora, se identifica la necesidad de acercarse a los mismos actores sociales que desarrollan estas prácticas; asimismo, como ya se mencionó, en la ciudad de Aguascalientes existen otras características sociodemográficas diferentes a las referidas en la literatura; también se observan diferencias en el tipo de participantes, por lo que las motivaciones o significados de estos individuos parte de otra necesidad o función. Es por ello que la pregunta de investigación que guía este estudio es "¿qué practicas económicas alternativas se desarrollan en la ciudad de Aguascalientes, y cómo dichas prácticas son significadas por sus participantes?"; esta pregunta también generó el siguiente objetivo: comprender qué significados otorgan los participantes a las prácticas de economías alternativas, e identificar cómo estos significados inciden en las prácti- 
cas, de modo que han permitido la construcción social de los espacios. El método que se eligió para este estudio es la teoría fundamentada (TF).

\section{Método}

El estudio se efectuó en la ciudad de Aguascalientes con dos diferentes grupos que desarrollaban actividades económicas alternativas como el tianguis o mercado alternativo y el espacio de trueque. La recolección de datos se hizo en un periodo de 11 meses. Se utilizaron distintas técnicas, como la observación participante en los lugares físicos, pero también ser parte de los grupos que ellos tenían en sus redes sociales (WhatsApp y Facebook) y ocho entrevistas semiestructuradas a informantes clave. La observación participante se hizo en distintos escenarios: 1) en parques donde se desarrolla usualmente la actividad; 2) en la calle Carranza, donde llegaron a converger ambos grupos promovidos por el Instituto Municipal Aguascalentense de la Cultura, y 3) en la casa donde hacían reuniones los participantes del trueque con algunos artesanos del estado. El número de entrevistas fue en función de la saturación teórica y de los datos obtenidos por la observación participante, esta última recomendada por Corbin (2010) como elemento importante para descubrir nuevos significados e intenciones, así como elemento de contraste o complementaria de la información obtenida en entrevistas.

Para la selección de los participantes se hizo un muestreo no probabilístico por conveniencia; ellos fueron identificados por medio de la observación participante como informantes clave, por ejemplo, coordinadores de los espacios de trueque y de los tianguis alternativos, así como a otros productores y participantes de esos espacios. Para la aplicación de las entrevistas se utilizó un guión temático encaminado a conocer las prácticas económicas y los significados que le otorgaban a la ejecución de éstas; no había categorías predefinidas; éstas se elaboraron conforme se analizaban los datos como marca la TF. Se obtuvieron los consentimientos informados y los avisos de privacidad; fueron grabadas y tuvieron una duración de entre 15 y 60 minutos. Se transcribieron utilizando el programa de windows media y la aplicación en computadora de Speechnotes.

A partir de las técnicas de recolección de datos comentados, se identificó que estos dos grupos, los que se desarrollan en la actividad del trueque y los que participan en el tianguis alternativo, de manera general están dentro de la clase media o media alta, la mayoría de los integrantes tiene estudios universitarios o está en un proceso de formación (preparatoria o universidad), par- ticiparon jóvenes y adultos como los principales actores, aunque también hubo inclusión en algunas actividades como los talleres a niños y adultos mayores.

Con el objetivo de que el análisis de los resultados tenga mayor claridad se dará una amplia explicación sobre la TF. Después se describe cómo fue utilizada en esta investigación.

\section{TEORÍA FUNDAMENTADA}

Para Glaser y Strauss (1967), autores de la Teoría fundamentada, no hay mejor manera de retratar la realidad social que hacerlo mediante la recogida sistemática de los datos en lugar de comprobar teorías ya existentes basadas en la especulación, por lo que la teoría que surja con la implementación de este método aumenta la posibilidad de comprensión del fenómeno y, por tanto, aporta una guía más relevante para la acción (Abela, García-Nieto y Pérez-Corbacho, 2007; Strauss y Corbin, 2002).

Se llama Teoría fundamentada o Teoría enraizada porque la teoría deviene de los mismos datos. Algunos de los aspectos que caracterizan a la TF es que es un método inductivo en el que la recogida de datos y el análisis de éstos se efectúa de manera simultánea, a diferencia de otros métodos que comienzan el análisis después de la obtención de los datos. Es por ello que no hay hipótesis a priori, pues éstas se generan durante el proceso. De ahí que se inicie con preguntas directrices.

Otra característica importante de la TF es la circularidad del proceso, porque hay una interacción constante entre la recogida de datos, el análisis de éstos (codificación), el surgimiento de nuevas preguntas y el regreso al campo para continuar recabando datos hasta llegar a la saturación (figura 1). En la TF hay distintos tipos de codificación y tipos de códigos.

\section{Codificación desde la Teoría fundamentada}

Para este proceso existen tres tipos de codificación y tres tipos de códigos. Los tipos de codificación son 1) abierta; 2) axial, y 3) selectiva; los códigos pueden ser 1) in vivo; 2) sustantivo, y 3) teórico. El primero alude al nombre dado por la persona entrevistada, el segundo a la descripción del fenómeno y el tercero al concepto que permite la comprensión del fenómeno. El proceso de codificación se da de manera circular, porque es común volver de modo recurrente a los procesos anteriores para verificar y modificar el código (Abela, García-Nieto y Pérez-Corbacho, 2007; Strauss y Corbin, 2002). 


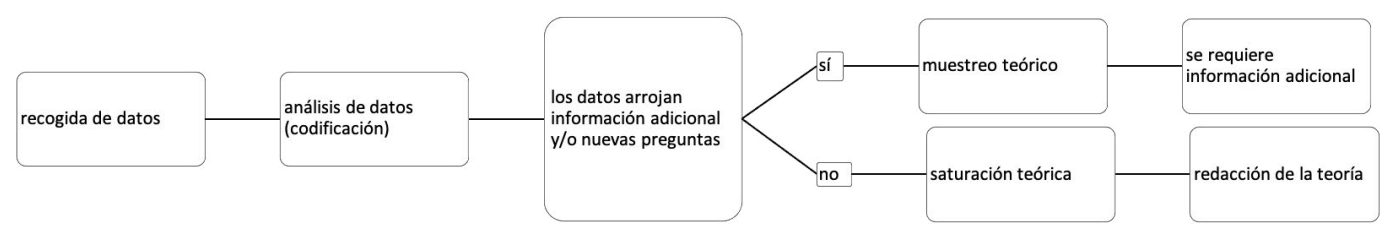

Figura 1.

Muestreo y saturación teórica.

\section{Codificación abierta}

Consiste en una lectura recursiva línea por línea o párrafo por párrafo para segmentar de manera minuciosa la información para identificar pensamientos, ideas o significados en la información. Una vez identificados los datos se hace una cita para seleccionar la parte del texto que contiene información relevante para la investigación con el objetivo de ser etiquetados (codificados) y comenzar a desarrollar descripciones o conceptos.

La TF busca que los códigos emerjan de los datos; sin embargo, existen también los códigos de "construcciones sociológicas" en los que se permite retomar el conocimiento previo del investigador que va recordando al leer la información contenida en los datos (Abela, García-Nieto y Pérez-Carbacho, 2007).

Los conceptos son la forma de nombrar el fenómeno y surgen por medio de impresiones, significados y pensamientos que emergen de los datos obtenidos: los conceptos que tienen relación con el fenómeno se denominan categorías, y uno de los pasos para identificarlas es cuestionarse "¿qué sucede aquí?". Los elementos subordinados a las categorías tienen la función de dar más detalles o elementos para ampliar la comprensión del fenómeno. Algunas preguntas que permiten identificarlas son cuándo, dónde, porqué y cómo es probable que ocurra el fenómeno.

\section{Codificación axial}

La codificación axial consiste en comenzar a unir los datos que se fragmentaron durante la codificación abierta; sin embargo, previamente es necesaria la elaboración de categorías con la finalidad de establecer relaciones entre ellas. Las categorías representan al fenómeno y pueden ser acontecimientos o los aspectos significativos para la persona entrevistada. Las subcategorías también surgen al contestar preguntas referentes a cuándo, dónde, por qué, quién y con qué frecuencia (Strauss y Corbin, 2002).

La codificación axial requiere que, además de las relaciones entre las categorías se ubiquen dentro de un contexto. A este proceso se le llama Paradigma de la Codificación.
Este permite entender por qué se presenta el fenómeno, cuáles circunstancias lo favorecen, cuáles lo mantienen o lo limitan. Además, permite identificar qué estructura tiene y cuál es el proceso (Strauss y Corbin, 2002).

Una de las maneras en que uno se puede dar cuenta de esto es respondiendo a la pregunta " $i A$ qué problemas responde?". Asimismo permite identificar las consecuencias de las prácticas a nivel micro y macrosocial, qué consecuencias tiene a nivel personal y grupal, y en un sector más amplio.

\section{Codificación selectiva}

Este tipo de codificación tiene como objetivo integrar y refinar la teoría. El investigador previamente pudo haber identificado y jerarquizado algunas categorías que parecían ser el eje conductor para comprender el fenómeno. Durante esta codificación se pretende identificar la categoría central. Ésta es la que tiene mayor número de relación con otras categorías y se encuentra repetidamente en los datos.

Una técnica utilizada para identificar la categoría central es escribir el argumento de la historia, además de recuperar los diagramas y memorandos utilizados durante el proceso de codificación. Los memorandos son las notas personales que el investigador hace en referencia a los hallazgos encontrados y a las posibles hipótesis formuladas por medio de identificar las relaciones entre los códigos. Además ayudan conocer cómo se fue desarrollando la construcción de un código, así como la descripción de cómo identificó las relaciones que determinaron la categoría central y las subcategorías (Strauss y Corbin, 2002).

Previo a la escritura de la teoría formal es necesario identificar el nivel de saturación teórica para asegurarse que la información recolectada es suficiente para la comprensión del fenómeno (Cuñat, 2007). Posterior a eso se redacta la teoría. Es conveniente mencionar que este es un proceso dinámico porque de manera constante se regresa a alguno de los tipos de codificación para mejorar los códigos, las categorías y las relaciones entre éstas. Como consecuencia de esta constante interacción 
se puede pasar a la redacción de la teoría o se vuelve al campo a recolectar más información que permita la comprensión del fenómeno.

\section{Procedimiento de análisis de los datos}

Las transcripciones de las entrevistas y los datos obtenidos de las publicaciones hechas en las redes sociales se analizaron mediante el programa Atlas-ti versión 7.5. El análisis se dividió en cuatro fases: 1) lectura de los datos; 2) codificación; 3) análisis de las relaciones entre códigos, y 4) redacción de la teoría (figura 2 ). Una vez añadidos los datos al Atlas-ti se prosiguió a efectuar la codificación abierta que consistió en el análisis del texto completo sin hacer anotaciones. Después se hizo un análisis párrafo por párrafo. Se leyó de modo minucioso el contenido de un párrafo para ser marcado como una cita y en seguida se etiquetó con un código (codificación abierta).

En la codificación axial se comenzaron a hacer rela-

\section{Resultados}

Con el objetivo de exponer los resultados de modo más claro se presentarán en dos apartados. En el primero, a manera de contextualización, se describen los hallazgos encontrados, las características de los espacios y los participantes. En el segundo se exponen las categorías que emergieron del análisis. Se integra una explicación contextual para que las categorías puedan ser comprendidas con mayor facilidad. Es necesario destacar que éstas están relacionadas entre sí, por lo que en el trabajo se mostrará la vinculación entre las categorías que se entrecruzan.

En la investigación se estudiaron los grupos que participaban en el tianguis o mercados alternativos y los que practicaban el trueque. Los primeros dedicados a actividades lucrativas y los segundos a actividades no lucrativas. Ambos grupos tenían una frecuencia semanal. En el tianguis alternativo suelen encontrarse pequeños productores que comercializan de manera directa

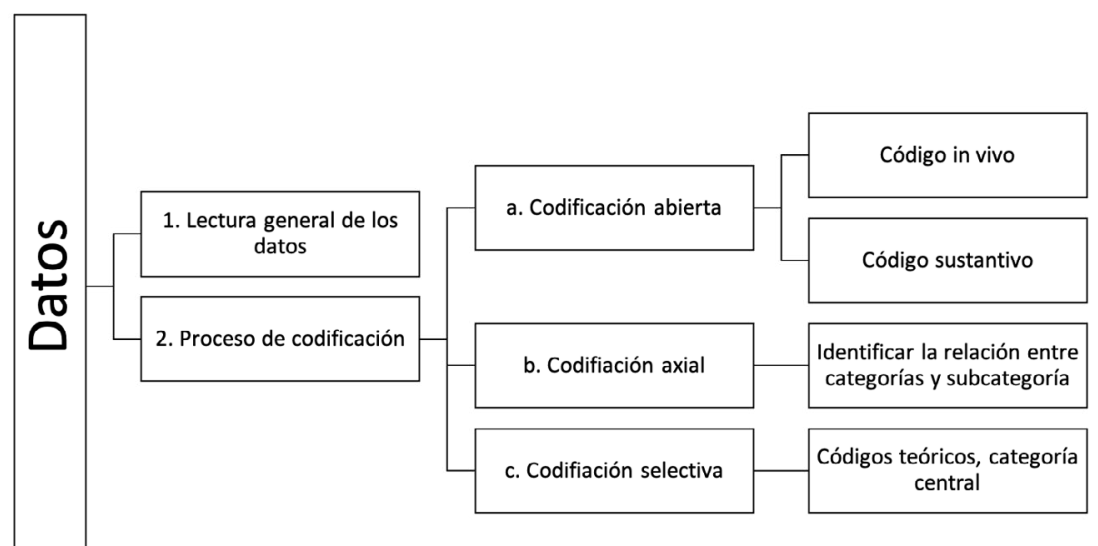

Figura 2.

Proceso para desarrollar teoría fundamentada.

ciones entre las categorías y se analizaron los elementos de éstas. Cuando las propiedades eran las mismas se fundieron en una sola categoría, y cuando las propiedades eran diferentes se identificaron relaciones o contradicciones entre ellas. Después se hizo una búsqueda de las categorías centrales (codificación selectiva). Éstas fueron evidentes por ser las más repetidas y con mayor alcance explicativo. En el proceso se elaboraron 215 códigos; a partir de las relaciones entre ellos resultaron cuatro categorías, las cuales se describen en el siguiente apartado.

Durante la recolección de datos se tuvo especial cuidado con el anonimato de las personas; por ejemplo, cuando se tomaban fotografías. Para hacer las entrevistas se entregó un formato de consentimiento informado y un aviso de privacidad; además, en las trascripciones no se utilizó su nombre y se cuidaron los datos personales que de alguna manera evidenciaran la identidad del informante. con el consumidor productos agroecológicos, comida vegana, productos ecológicos para la limpieza del hogar o el aseo personal, cosmética natural, café orgánico, artesanías, reiki y terapia floral. Se promueve la venta de artículos de uso cotidiano reutilizables como termos o popotes de acero inoxidable. De igual modo se pueden encontrar productos relativos al ecofeminismo y artículos y servicios para mascotas.

En los espacios en que la actividad principal es el trueque se encuentra el intercambio de artículos; cada fin de semana los participantes llevan los objetos que ya no son de su interés y hacen el intercambio con otro asistente. En ocasiones se usa una moneda social para organizar el ejercicio, sobre todo cuando la cantidad de participantes es numerosa. Los trueques se pueden pactar desde las redes sociales para ofertar sus objetos y 
hacer acuerdos con otros miembros del grupo (sin necesidad de ir de modo personal al lugar habitual).

En ambos espacios se promueven foros y talleres. Las pláticas versan acerca de diferentes temáticas, por ejemplo, cómo hacer composta, huertos orgánicos, reproducir plantas, economía o restauración de libros. Se dejan espacios abiertos para que los miembros compartan sus saberes o habilidades, así como para aceptar la participación de personas externas al mismo.

Una cuestión particular que se identificó en la investigación es que los sujetos que suelen estar involucrados en economías alternativas dentro de la ciudad de Aguascalientes son personas con estudios superiores, es decir, suelen tener una formación profesional. Además, es común que participen en varios de estos mismos espacios, es decir, si alguno de ellos vende comida vegana, ofrece sus productos en mercados alternativos en diferentes lugares.

Otra característica de estos participantes es que también tienen alguna otra fuente de ingresos, por ejemplo, los que tienen formación profesional tienen un empleo relacionado con su carrera, pero participan en estos tianguis o lugares de trueque los fines de semana. Se identificó que la principal fuente de ingresos de estos participantes no proviene de estos espacios, pero sí satisface otras necesidades. Los espacios tienen una función importante en la expresión ideológica de sus miembros, algunos autonombrados a sí mismos como activistas por la manera en que consumen determinados productos y promueven algunas prácticas (cuidado del medio ambiente, rescate de animales, ingerir comida vegana o vegetariana).

Con base en lo anterior y para concretar cómo tienen lugar los significados y las prácticas para los participantes, para ellos estos espacios son una manera de expresar, fomentar y compartir estilos de vida que deviene de una adhesión ideológica, de modo que los artículos aquí promovidos son diferenciados del mercado convencional, desde la forma de producción, elaboración y distribución. Asimismo, por medio de sus prácticas intentan concientizar acerca del sentido original del trabajo, así como priorizar la calidad de vida respecto a la acumulación. Se observa que lo que motiva a estos participantes en Aguascalientes a integrarse a estos espacios es el activismo por el cuidado al medio ambiente y la promoción de un estilo de vida que promueva la práctica de otras formas de consumo y de relación con el medio ambiente; así, estos participantes distan de los comúnmente reportados en la literatura como pertenecientes a un grupo vulnerable, además de que se prioriza más el cuidado de la relación que se tiene del medio ambiente, más que ser una opción para obtener como primera fuente de ingre- sos el solventar las necesidades básicas. Igualmente debe considerarse que los significados y las intenciones no son estáticas, sino que se definen y se redefinen mediante las diversas interacciones en y fuera del espacio.

Por último se identificó que se pueden utilizar de manera indistinta los términos "economía social", "economía solidaria" y "economía circular". Usar estos términos posibilita que los participantes tengan acceso a los espacios públicos donde puedan desarrollar sus actividades, por lo que también estas etiquetas son utilizadas de manera instrumental, pues denominarse dentro de estas etiquetas facilita que les den espacios en parques y jardines, así como en algunos otros lugares públicos. A pesar de que el nombre del tipo de economía puede cambiar según el acceso a los espacios, lo que también se observa es que escogen estas etiquetas porque tienen una ideología afín al cuidado animal y ambiental. Para los participantes es significativo contar con lugares donde puedan ingresar los pequeños productores y activistas con el objetivo de ganar espacio público y político.

Una vez hecha la contextualización se presentan las categorías. Durante el proceso de análisis de datos que incluyó la codificación abierta, axial y selectiva se identificaron cuatro categorías centrales: 1) alternativo; 2) perfil de quien participa, 3) ideología, y 4) activismo. A continuación se explicará cada una de ellas.

\section{Alternativo}

Esta categoría se refiere al conjunto de prácticas económicas diferenciadas de los participantes, donde denotan una posición ideológica ante el sector económico dominante, un modo de producción distinto y el perfil de quienes participan en dichas actividades. Estos espacios suelen autonombrarse como alternativos porque representan una opción frente a otros tipos de mercados, como los mercados capitalistas. Lo alternativo se distingue en dos sentidos: las prácticas y el componente ideológico; la primera se refiere a la acción desarrollada por los participantes, mientras que la segunda hace referencia al significado, la intención o postura ideológica que motiva a la práctica.

[...] Una diferencia quizá de las más importantes de la economía solidaría y el capitalismo es que en la economía solidaria lo más importante es la persona, ¿no? Por eso yo te digo que en el trueque nuestra finalidad sería que pudiéramos satisfacer nuestras necesidades como personas y crecer como personas; por eso se invita a los chicos a hacer estos productos para que ellos tengan una herramienta en el futuro [...](María).

[...]Si lo ves es muy diferente a cualquier otra expo, ¿por qué?, porque en una expo vas, y vas a ver qué com- 
pras, y aquí hacen un super, o sea, aquí es un cambio de conciencia; todos los productos que entran aquí tiene que demostrar que son agroecológicos, no hay nada con pesticidas, sobre todo lo que es comida; tratamos también de los demás productos que sean todos amigables con el medio ambiente, que sean sustentables y que den algo diferente, un plus, no nada más sea un producto por ponerte y ya [...](Romina).

Una vez más aparece el componente ideológico, el cambio de conciencia como lo que posiciona a estos sujetos como seres alternativos, pues llevan prácticas con fines de tener una vida digna y sustentable protegiendo al medio ambiente.

[...] tenemos otro tipo de necesidades como seres humanos, no sólo materiales, espiritual, social, cultural; este, y esa parte el mercado convencional que es primordialmente capitalista no lo ve, no es importante, no es lo relevante, ¿no? En estos mercados sí; es justo que lo queremos, lo que buscamos; le damos más importancia al bienestar de las personas (Sofía).

Como ya se mencionó, las prácticas son varias; sin embargo, son en búsqueda de acciones que promuevan formas alternativas que satisfagan las necesidades de sus usuarios, no sólo en bienes materiales sino relacionales, ideológicos, donde son aceptados por el consenso social; es decir, el desarrollo de estas prácticas tiene sentido en ese espacio, dada la finalidad del mismo; no obstante, el costo de los productos es más elevado que los ofertados en un tianguis popular, por lo que el productor y el consumidor usualmente comparten características o perfiles.

\section{Perfil de quien participa}

Esta categoría hace referencia a las formas de autonombrarse y diferenciarse según el tipo de actividad o rol desempeñado dentro de estos espacios, y en ocasiones también desempeñados fuera de este escenario. Los participantes buscan ser reconocidos como alternativos mediante prácticas diferenciadas a las ofrecidas en el mercado dominante. Esto lo consiguen por medio de la manifestación ideológica a la que son afines en ese espacio, adoptando estilos de vida diversos; éstos se ven matizados en una amplia gama de perfiles. Ellos suelen autodenominarse "rescatistas", "ecologistas", "ambientalistas", "antiespecistas", "pequeños productores", "veganos", prosumidores", "traidores", etcétera. Esta adhesión ideológica configura algunos patrones de comportamiento que pueden ser o no constantes en el tiempo, pero que desempeñan un papel importante dentro de ese espacio.

[...] he identificado personas afines con intereses comunes; si las ves, es difícil que alguna no sea rescatista; todas son ambientalistas, son ecologistas; todas las perso- nas que participan en esto, la mayoría de ellos no tienen fecha; me habla de una congruencia, ¿sí? Yo sí tengo; no disponer de un vehículo contaminante (Raúl).

Estas formas de autonombrarse también marcan diferencias entre los participantes, pues también definen el propósito o significado de su actividad. Si bien ellos mismos pueden sentirse identificados con más de una categoría, también les permite identificarse como personas "congruentes" en la relación entre intenciones y prácticas efectuadas. La intencionalidad de la práctica también es un elemento importante o que marca distinción en estos espacios, pues ellos identifican que algunos participantes utilizan el espacio y las etiquetas de manera instrumental:

[...] no era su forma habitual de vestir, no me agradó; me pareció un fingimiento y una técnica de venta. Cuando dejas de ser auténtico, como que cuando necesitas esconderte, o disimular, ya puedes dudar de otras acciones (Raúl).

Además, las etiquetas también muestran formas de radicalidad ideológica, pues para ellos denota qué tan comprometido o congruente eres ante dicha causa; es ahí donde se observa la diferencia entre el grado de activismo y adhesión ideológica.

\section{Ideología y activismo}

Esta categoría originalmente se planteó como dos categorías independientes, es decir, a) ideología, y b) activismo; sin embargo se decidió integrar en una sola porque guardan una estrecha relación entre sí: para el estudio esta categoría se define como "ideología", hace referencia al conjunto de creencias, posicionamiento, ideas que disponen formas de actuar; mientras que "activismo" es una práctica que pretende demostrar una posición ideológica o defender una causa política, social, ambiental. Aquí el tipo de práctica específica y los significados tienen una función destacada respecto al nivel de activismo y a la flexibilidad o radicalidad de la adhesión ideológica.

Se identificó que las actividades lucrativas implementadas en el tianguis, si bien tienen la intención de poner en práctica el activismo mediante el tipo de productos que venden, existe mayor margen de flexibilidad y permiten la convivencia con personas que no comparten la misma ideología, mientras que el grupo que desarrollaba actividades no lucrativas haciendo trueque, mostraban menos flexibilidad y menos permisividad para efectuar actividades en contra de los códigos morales establecidos en el mismo grupo, como no permitir el consumo de alimentos provenientes de animales mientras se llevaba a cabo el intercambio o abandonar espacios de venta porque se permitía la venta de cárnicos. 
[...] decidí salirme porque la organizadora me dijo que era vegana y permitía la venta de carne de conejo. A veces la ética tiene precio. Sé que la situación está difícil, pero yo prefiero ser lo menos incongruente posible que tener dinero (Ximena).

Si bien se pueden encontrar estas posiciones dicotómicas, también aquellos que se muestran más flexibles lo hacen con la finalidad de que poco a poco los miembros que participan, aunque no tienen una adhesión ideológica regida por todos los códigos éticos y morales impuestos en los grupos, promuevan la participación por medio de diferentes estrategias como los talleres, cursos de inducción, foros, la manera en la que solicitan que se comercialice el producto al consumidor, así como permitir la participación en el espacio.

[...] tampoco se les puede obligar. Porque no es nuestra intención excluir a las personas; al contrario, ¿cómo vamos a involucrarlos en estas dinámicas si nunca lo ven, si nunca lo palpan, lo experimentan de menos con el vecino que tal vez si lo está haciendo?, ¿no? (Sofía).

\section{Discusión}

Los productos típicos ofertados en los mercados alternativos de la ciudad de Aguascalientes son productos agroecológicos, ecológicos, orgánicos y artesanales, entre otros (Roldán et al., 2016). Este tipo de oferta mercantil es lo que los distingue de otros espacios y otros supermercados o tianguis populares. En estos espacios se difunde el prosumo, es decir, ser productor y consumidor de los mismos compañeros del mercado o tianguis. Se observa que el componente adicional de estas mercancías, es decir su valor agregado, es que son productos alternativos que mejoran la salud y preservan el medio ambiente; además, como lo indican Duhau y Giglia (2007), las prácticas de consumo están motivadas por componentes idiosincráticos y socioespaciales, por lo que tanto productores como consumidores acuden a este tipo de espacios por una ideología compartida.

A diferencia de la población referida en la literatura, se observa que en Aguascalientes los actores involucrados en estos espacios no se encuentran en alguna situación de exclusión o marginación social, pero son un grupo minoritario dentro del contexto histórico cultural de dicha ciudad. Un ejemplo de ello son los grupos de activistas afines al feminismo, cuya ideología les hace reconocer algunos principios de las economías alternativas como congruentes a los fundamentos del feminismo (Coutinho, Beiras, Picinin y Lückmann, 2005; Calil y Szymanski, 2010; Marañón-Pimentel, 2013; García y Valdés, 2015; Méndez, 2015; Santos et al., 2016).
Participar en estos espacios brinda a sus miembros una visibilidad política porque a partir de las interacciones ahí efectuadas hay un intercambio ideológico. Los grupos minoritarios, como los pequeños productores o artesanos, tienen la posibilidad de hacer resistencia ante el mercado dominante. Se observa que estos espacios pueden tener una doble funcionalidad; por un lado, se satisfacen las necesidades básicas, y por otro existe una instrumentalización de los espacios para el esparcimiento ideológico del que forman parte. Los sujetos que se adhieren a las corrientes de pensamiento presentes en estos espacios pueden ser nombrados ambientalistas, rescatistas, antiespecistas, feministas, prosumidores, ecologistas, veganos y vegetarianos, entre otros (Roldán et al., 2016).

Otro aspecto que no se evidencia en los estudios anteriores es la perspectiva dicotómica que surge en estos espacios, es decir, al definirse como ambientalistas, prosumidores, feministas, o alguna otra manera de las mencionadas, aquellas personas o grupos que no compartan su visión o estilo de vida son vistos como adversarios, personas faltas de conciencia y como responsables de que no haya un cambio en las condiciones sociales y ambientales que hoy se viven.

Como lo indican Sanz, Carrero y Alcañiz (2018), una pieza fundamental de la participación de estos espacios de economías alternativas son los discursos ideológicos de tipo político-moral que son el punto central de la diferencia entre lo convencional y lo alternativo (economías alternativas versus capitalismo). Mediante estos discursos se promueve entre los clientes el consumo responsable sin incurrir en daño ambiental o animal, además de no contribuir a la explotación de los trabajadores ni promover la acumulación. Se identificó la importancia que prevalece en estos espacios la posición ideológica, pues para varios de ellos participar en estos espacios significa una forma de activismo.

En los diferentes procesos de codificación, sobre todo en la codificación abierta, fue evidente que, si bien para los participantes las economías solidarias o alternativas buscan privilegiar al hombre sobre el capital, también hay una connotación de activismo ambiental y proanimal sobre un interés filantrópico. Si bien el antiespecismo se escucha en sus discursos, se observa que las principales motivaciones están dirigidas a la preservación del medio ambiente y las especies sobre la búsqueda de la satisfacción de las necesidades humanas. Este activismo es más evidente dentro del espacio de trueque que del mercado alternativo.

Por otra parte, de acuerdo con Monzón-Campos (2006) y Pérez-Mendiguren, Etxezarreta-Etxarri y Guridi-Aldanondo (2008), en estos espacios se privilegia la partici- 
pación de las mujeres, así como buscar formas alternativas para una vida digna y sustentable. Se identificó que, si bien las mujeres eran en muchas ocasiones las coordinadoras o promotoras de estas actividades, no era un elemento que los participantes destacaran en sus discursos. No obstante, un aspecto que sí se vislumbraba en algunos de ellos era el contraste entre el capitalismo y el feminismo, y cómo este último se vincula con las economías solidarias.

Los aspectos identificados al implementar este método, en el que se teoriza a partir de los datos obtenidos y no desde teorías prestablecidas (Strauss y Corbin, 2002), contribuyeron a comprender cómo surgen estas prácticas alternativas, a qué necesidades responden y cómo los significados incidían en dichas prácticas en Aguascalientes. Estos datos tienen contrastes significativos a los reportados en la literatura en cuanto a las características de los participantes, así como el evidente carácter ideológico que motiva el desarrollo de las prácticas en estos escenarios. Respecto a las limitaciones encontradas en el estudio, se tuvo dificultad para conversar con varios productores, porque para hacer las entrevistas sólo accedían hacerlas dentro del mismo espacio de venta, por lo que con frecuencia había interrupciones y en ocasiones no había oportunidad de profundizar acerca de los significados con algunos de ellos; asimismo, la elaboración de las categorías fue un proceso largo porque éstas se formaban y adaptaban conforme se obtenían los datos; además fue necesario hacer adaptaciones al método porque no se podía analizar una entrevista y volver a la colecta de datos debido a la poca disponibilidad de tiempo de los participantes; algunas entrevistas se hacían en conjunto para su posterior análisis, aunque, según Strauss y Corbin, (2002), sólo dan algunas directrices para realizar TF, pues es un método flexible.

\section{Conclusiones}

Respecto al objetivo de la investigación acerca de los significados y las implicaciones que tienen en sus prácticas económicas se identificó que, si bien el trueque puede ser visto como una práctica precapitalista, el resurgimiento de esta práctica en este espacio significó para los participantes una alternativa para ampliar la vida útil de los objetos, y con ello disminuir el impacto ambiental, además de solventar alguna necesidad prescindiendo del dinero para el intercambio, lo que también es significativo para ellos porque les permite experimentar que se abren posibilidades ante el mercado dominante.

Asimismo, los significados de las prácticas económicas alternativas radicaron en 1) otras formas de producción, donde se optaba por la elaboración de productos agroecológicos y los productores tenían que someterse a un escrutinio interno por parte de los coordinadores del tianguis para comprobarlo; 2) lo diferente en la presentación del producto y forma de venta, donde se tiene contacto directo entre el productor y consumidor, por lo que se facilita promover y compartir una ideología, y 3) el tipo de productos, usualmente más dirigidos a lo artesanal o de preparación lenta, así como la promesa de tener un bajo impacto ambiental.

Asimismo se identificó que el autonombrarse como "economías solidarias", si bien puede usarse de modo instrumental, también es apropiado como un componente ideológico donde pueden poner de manifiesto expresiones de activismo, donde se buscan formas alternativas de producción y satisfacción de necesidades materiales, sociales y relacionales donde se promueven prácticas de consumo diferenciadas al mercado dominante.

Por otro lado, durante la investigación las limitaciones encontradas fueron que es un espacio excluyente no sólo para los que piensan diferente, sino que también para personas de clase baja, ya que estos espacios tienen un costo más elevado al mercado convencional o los tianguis populares; en expresión de un consumidor, son elitistas, porque no son costos accesibles, por lo que el cuidado ambiental o la salud no están al alcance de todos.

Respecto al desarrollo metodológico, se identificó como una virtud el haber implementado TF porque permitió ampliar el nivel de comprensión de este fenómeno, así como adentrare en los significados otorgados de los participantes. La diversidad de técnicas de recolección de datos, como entrevistas, observación participante y la información publicada por los participantes en las redes sociales, permitió notar los contrastes y similitudes entre lo dicho en el discurso y sus prácticas.

Por otro lado, ya que al implementar teoría fundamentada se sugiere que la teoría surja de los propios datos, así como ingresar al campo sin hipótesis, la recolecta de datos y el dar un orden a ellos requiere soportar altas dosis de ambigüedad y el no tener claridad acerca de la relevancia o no de algunos datos. Asimismo, la dificultad de integrar la información recabada y de analizar cada dato antes de hacer nueva colecta de los mismos, en ocasiones es poco práctico o inviable dadas las condiciones en que se hizo la investigación.

Para finalizar, hemos identificando que el artículo se concluye en un contexto mundial de emergencia sanitaria por el nuevo coronavirus Sars-CoV-2, y dados los estragos en cuestiones de salud, sociales y económicas, sería interesante investigar si este tipo de prácticas económicas alternativas permanecen o surgen en nuevos espacios y con distintas poblaciones e identificar qué alcances y limitaciones tienen al adaptarlas. Se sugiere 
que en próximas investigaciones también se aborden estostos desde distintos marcos teóricos o métodos, como la investigación-acción.

\section{Referencias}

Abela, A. J., García-Nieto, A., \& Pérez-Corbacho, A. M. (2007). Evolución de la teoría fundamentada como técnica de análisis cualitativo (1a. ed). Madrid, Centro de Investigaciones Sociológicas.

Avendaño, O., Canales, M., \& Atria, R. (2012): Sociología: Introducción a los clásicos. K. Marx, E. Durkheim, M. Weber. Santiago, Lom Ediciones.

Calii, S. D., \& Szymanski, H. (2010). A constituição de uma cooperativa eo proceso identitário. Psico, 41(3), 356-365.

Corbin, J. (2010). La investigación en la teoría fundamentada como medio para generar conocimiento profesional. En S. Bernard (ed.). La teoría fundamentada. Una metodología cualitativa (pp.13-54). México, Universidad Autónoma de Aguascalientes.

Coutinho, M. C., Beiras, A., Picinin, D., \& Lückmann, G. L. (2005). Novos caminhos, cooperação e solidariedade: A psicologia em empreendimentos solidários. Psicologia \& Sociedade, 17(1), 7-13.

Cuñat, R. (2007). Aplicación de la teoría fundamentada (Grounded Theory) al estudio del proceso de creación de empresas. XX Congreso anual de AEDEM, Decisiones basadas en el conocimiento y en el papel social de la empresa. Madrid, España.

Diez, M. A. (2002). ¿Qué son las llamadas economías alternativas? Millcayac. Anuario de Ciencias Políticas y Sociales, 1(1), 205-266.

Duhau, E., \& Giglia, A. (2007). Nuevas centralidades y prácticas de consumo en la Ciudad de México: Del microcomercio al hipermercado. EURE, 33(98), 77-95. https://dx.doi. org/10.4067/S0250-71612007000100005.

García, F., \& Valdés, E. (2015). Producción de subjetividades en emprendimientos económicos solidarios. Una comparación de dos casos. Revista cooperativismo y desarrollo, 5(12), 28-38.

Glaser, B., \& Strauss, A. (1967). The discovery of grounded theory. Londres, Weidenfield \& Nicolson.
González, A. A. (2011). Mercados alternativos locales frente al sistema agroalimentario global. Líder, 1 (19), 39-56.

Marañón-Pimentel, B. (ed.) (2013). La economía solidaria en México (1a. ed.). México, Universidad Nacional Autónoma de México.

Méndez, R. (2015). Redes de colaboración y economía alternativa para la resiliencia urbana. Una agenda de investigación. Revista Bibliográfica de Geografía y Ciencias Sociales, 20(1.139), 1-24.

Monzón-Campos, J. L. (2006). Economía social y conceptos afines. Fronteras borrosas y ambigüedades conceptuales del Tercer Sector. CIRIEC-España, Revista de Economía Pública, Social y Cooperativa, (56), 9-24.

Pérez-Mendiguren, J. C., Etxezarreta-Etxarri, E., \& GuridiAldanondo, L. (2008). ¿De qué hablamos cuando hablamos de Economía Social y Solidaria? Concepto y nociones afines. Comunicación presentada a las XI Jornadas de Economía Crítica, 27-29.

Roldán, H. N, Gracia, M. A., Santana, M. E., \& Horbath, J. E. (2016). Los mercados orgánicos en México como escenarios de construcción social de alternativas. Polis, 15(43), 1-18.

Santana, M. (2011). Los mercados alternativos y la economía solidaria. CAOS Revista Electrónica de Ciencias Sociales, 1(17), 136-146.

Santos, C., Monteiro, É.., Possamai, H., Giombelli, J., Pilati, L. H., Schmitz Albuquerque, M., Ampese, R., \& Machado Bazzi, V. (2016). Psicologia \& economia solidária: Possíveis intervenções. Diálogo (31), 47-57. http://dx.doi. org/10.18316/2238-9024.16.23.

Sanz, J., Carrero, G., \& Alcañiz, G. (2018). Retos metodológicos y potencialidades de la etnografía para el estudio de la Economía Social y Solidaria. En E. Santamaría, L. C. Yufra y J. Haba (eds.). Investigado Economías Solidarias (pp. 31 41). Barcelona, Associació ERAPI.

Strauss, A. L., \& Corbin, J. (2002). Bases de la investigación cualitativa: Técnicas y procedimientos para desarrollar la teoría fundamentada. Colombia, Universidad de Antioquía. 


\section{Meta-Análisis del Artículo}




\section{Dimensión Cuantitativa}

\section{Perfil de Evaluación entre pares}
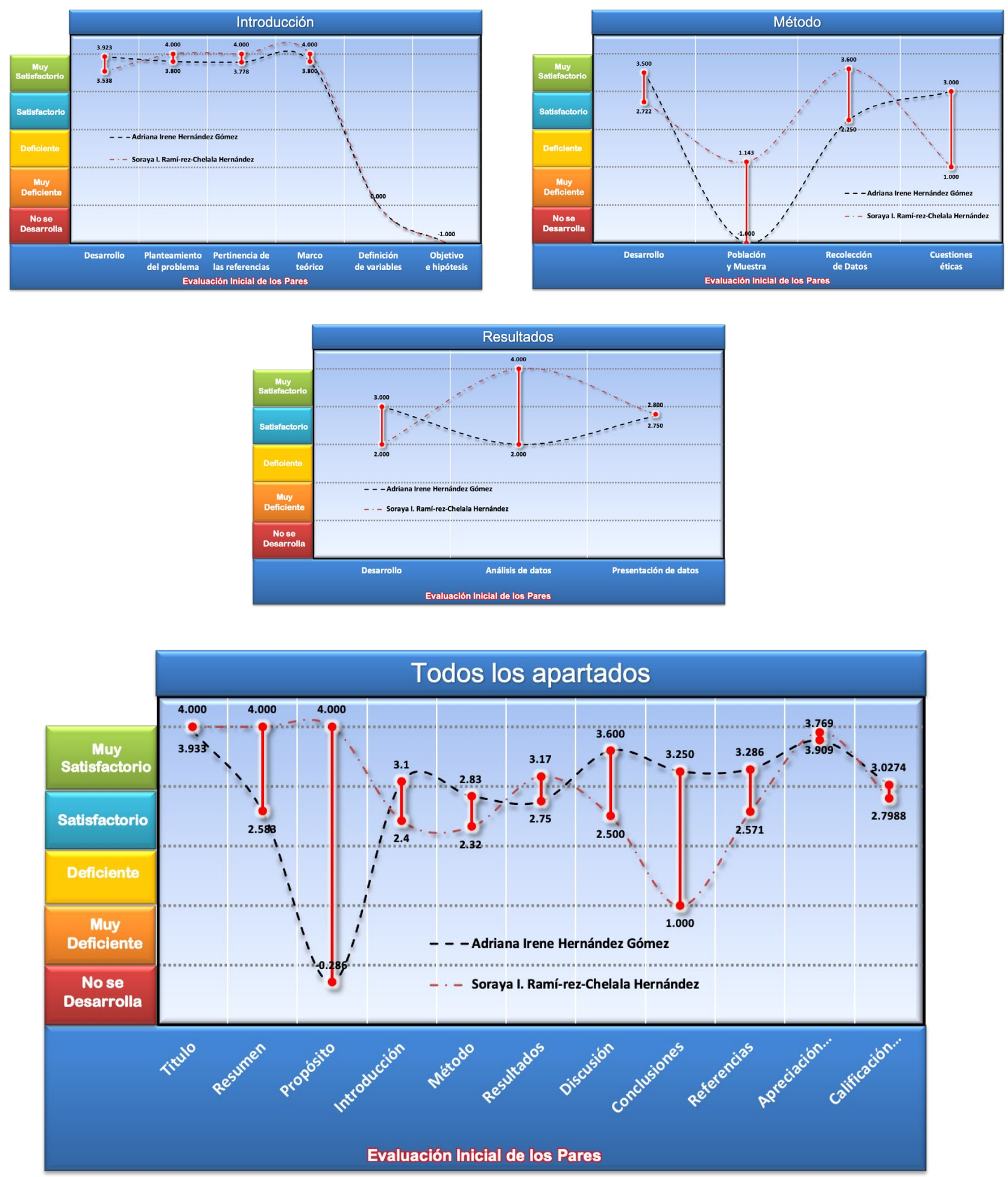


\section{Índice de Concordancia}

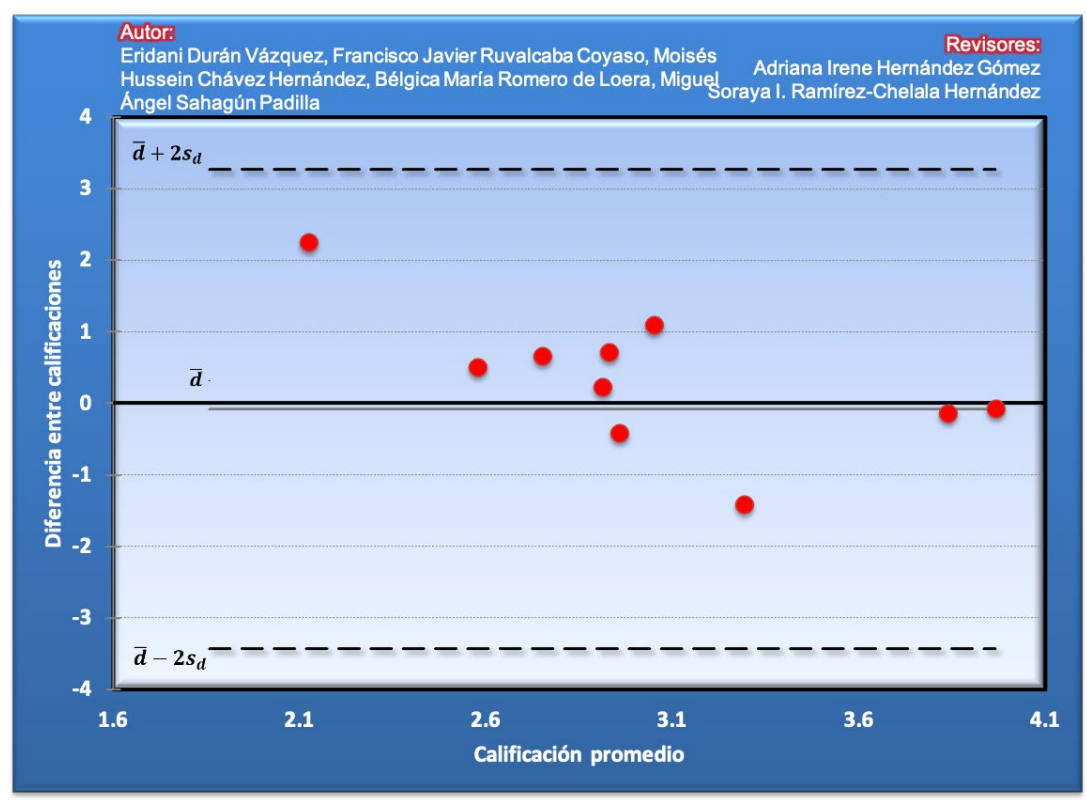

\section{Índice de Acuerdo}

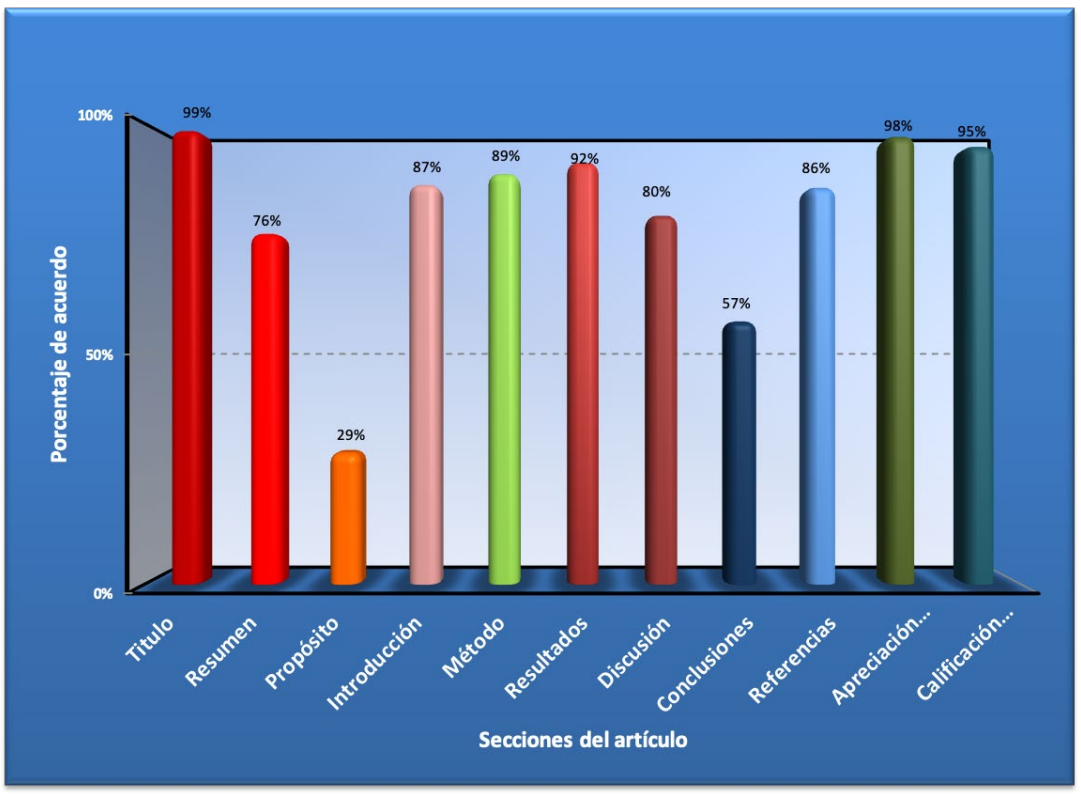




\begin{tabular}{|c|c|}
\hline Revisor 1 & Revisor 2 \\
\hline Adriana Irene Hernández Gómez & Soraya I. Ramírez-Chelala Hernández \\
\hline \multicolumn{2}{|c|}{ Título/Autoría } \\
\hline & Sin comentarios \\
\hline \multicolumn{2}{|c|}{ Resumen } \\
\hline & No aplica \\
\hline \multicolumn{2}{|c|}{ Próposito del Estudio } \\
\hline & $\begin{array}{l}\text { En la pregunta de investigación dice ¿qué practicas alter- } \\
\text { nativas se llevan a cabo en el estado de Aguascalientes y } \\
\text { cómo estas prácticas son significadas por sus participan- } \\
\text { tes? Hay que ser claros en cuanto a prácticas alternativas } \\
\text { en qué y significativas en qué sentido. }\end{array}$ \\
\hline \multicolumn{2}{|c|}{ Introducción } \\
\hline $\begin{array}{l}\text { En cuanto al Marco teórico está muy bien escrito y estruc- } \\
\text { turado; sin embargo, se afirma que hay poca literatura en } \\
\text { el área. Recomiendo los trabajos de la Dra. Angela Giglia, } \\
\text { quien investiga el tema desde hace años y es un referente } \\
\text { importante en la temática. No se enuncia el objetivo de la } \\
\text { investigación. Y aun cuando la investigación cualitativa se } \\
\text { centra sobre la pregunta de investigación (y ésta está bien } \\
\text { redactada), es importante incluir el propósito de la inves- } \\
\text { tigación. Hace falta también en el resumen. }\end{array}$ & $\begin{array}{l}\text { Desarrollar los objetivos y ponerlos en una apartado cla- } \\
\text { ramente identificables. }\end{array}$ \\
\hline \multicolumn{2}{|c|}{ Método } \\
\hline $\begin{array}{l}\text { El método carece de secciones, se sugiere agregarlas y } \\
\text { hacer un acomodo de la información en las mismas. Por } \\
\text { otro lado, en cuanto a los participantes del estudio, es- } \\
\text { tos no se describen. Pensando que es una investigación } \\
\text { cualitativa, la adecuada descripción de los participantes } \\
\text { y sus condiciones sociales, económicas, etáreas, etc., se } \\
\text { vuelve sustancial en el análisis de los resultados. Por otro } \\
\text { lado, no se justifica, ni se describe suficientemente el por } \\
\text { qué de las técnicas aplicadas. Esto es fundamental. Se re- } \\
\text { comienda reestructurar este apartado. }\end{array}$ & $\begin{array}{l}\text { Que en un apartado se diga el total de participantes que } \\
\text { integran los grupos y las características de esos grupos. }\end{array}$ \\
\hline
\end{tabular}




\begin{tabular}{|c|c|}
\hline Revisor 1 & Revisor 2 \\
\hline \multicolumn{2}{|c|}{ Resultados } \\
\hline $\begin{array}{l}\text { Los Resultados se quedan únicamente a un nivel descrip- } \\
\text { tivo, no únicamente la primera parte de los resultados, } \\
\text { sino también la segunda donde se presentan las catego- } \\
\text { rías. Por otro lado, los resultados no integran completa- } \\
\text { mente lo que establece la pregunta de investigación. Y } \\
\text { aunque la última categoría "Alternativo", pudiera tratar de } \\
\text { responder, al ser tan concisa, no lo expresa. Se recomienda } \\
\text { enriquecer el apartado de resultados. }\end{array}$ & $\begin{array}{l}\text { Los apartados de análisis de datos va antes de los resulta- } \\
\text { dos. Ya después discusión y resultados. }\end{array}$ \\
\hline \multicolumn{2}{|c|}{ Discusión } \\
\hline $\begin{array}{l}\text { Es una buena discusión; sin embargo, es importante } \\
\text { nombrar las limitaciones del estudio. Igualmente que } \\
\text { en los resultados, se toca de manera en extremo some- } \\
\text { ra la cuestión de cómo significan sus prácticas los par- } \\
\text { ticipantes. Una pequeña extensión en ambos sentidos } \\
\text { puede mejorar significativamente esta parte. }\end{array}$ & Se incluyen algunos comentarios \\
\hline \multicolumn{2}{|c|}{ Conclusiones } \\
\hline $\begin{array}{l}\text { Es importante ampliar las conclusiones en relación con } \\
\text { los propósitos de la investigación. Pero también es indis- } \\
\text { pensable abordar las posibles deficiencias o limitaciones } \\
\text { del estudio, ya que tampoco se abordan en la Discusión. } \\
\text { Se recomienda ampliar las Conclusiones en este sentido } \\
\text { y tal vez para no ampliar demasiado la extensión del do- } \\
\text { cumento, prescindir de la explicación de la Teoría Fun- } \\
\text { damentada que se encuentra en el Método, haciendo de } \\
\text { esta explicación sólo un párrafo que deje espacio al en- } \\
\text { riquecimiento de resultados, discusión y conclusiones. }\end{array}$ & $\begin{array}{l}\text { Revisar comentarios. Incluir objetivos para poder con- } \\
\text { trastar con resultados }\end{array}$ \\
\hline \multicolumn{2}{|c|}{ Referencias } \\
\hline $\begin{array}{l}\text { Es importante revisar el aparato crítico del documento. } \\
\text { No aparecen en el artículo los siguientes autores: Andréu } \\
\text { (2007), Giménez (2007), Haernecker, 1985) y Kormilov } \\
\text { (1924) En el cuerpo del texto aparecen varios autores } \\
\text { que no se incluyen en el listado de referencias: Szymanki } \\
\text { (2010), Santana (2011) y Avendaño (2012) En el texto se } \\
\text { cita a Diez (2002) y en el listado se coloca el año } 2003 \text {. }\end{array}$ & Revisar referencias \\
\hline
\end{tabular}




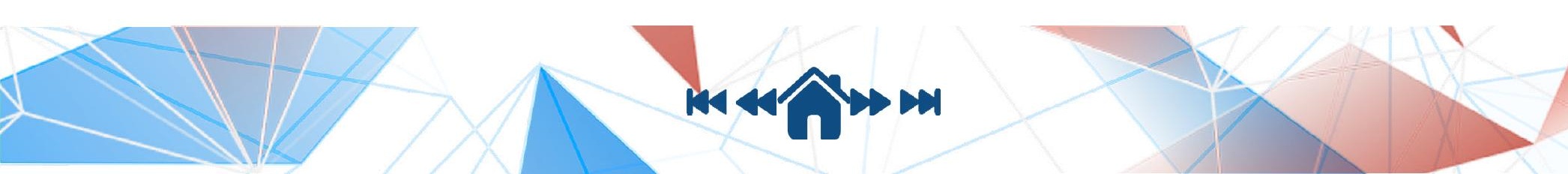

